\title{
An Axiomatics for Hyperbolic Projective-Metric Planes in Terms of Lines and Orthogonality
}

by

\author{
Victor PAMBUCCIAN
}

Presented by Czestaw BESSAGA

Summary. Hyperbolic projective-metric planes, first axiomatized by R. Lingenberg [7], are shown to be axiomatizable in terms of lines and orthogonality.

In a projective plane endowed with a hyperbolic projective polarity, one can define an orthogonality relation on the set of lines which are not selfpolar (i.e. not passing through their poles), two lines being orthogonal if they pass through each other's pole. The set of non-self-polar lines, together with the line-concurrence (three lines having a point in common are called concurrent) and orthogonality relations form a hyperbolic projective-metric plane (called a hyperbolic-metric plane in [7]). The motion group of this class of structures was first axiomatized in $[1, \S 11]$, and the structures themselves were first axiomatized in [7] (see also [6]) in a mixed language, containing on the one hand line-reflections, and on the other the incidence and orthogonality structure defined in terms of line-reflections.

The aim of this paper is to show that one can eliminate both the linereflection and the line-concurrence notions from Lingenberg's axiom system, to obtain an axiom system for hyperbolic projective-metric planes with lines as the only individual variables and line-orthogonality as the only primitive notion.

2000 Mathematics Subject Classification: 51M10, 51F15, 51F20, 51F25, $03 \mathrm{~B} 30$.

Key words and phrases: hyperbolic projective-metric plane.

This paper was written while the author was at the University of Białystok with a Fulbright grant. I thank both the Polish-U.S. Fulbright Commission for the grant and the Institute of Mathematics of the University of Białystok, and in particular Professor Krzysztof Prażmowski, for the hospitality. 
It thus turns out that hyperbolic projective-metric planes (both over arbitrary fields of characteristic $\neq 2$ and over quadratically closed fields of characteristic $\neq 2$ ) can be axiomatized in the same language as plane hyperbolic geometry (cf. [11]-[14]), and can be treated as a Plücker space, as defined in [2].

Our result can also be stated in the spirit of [3]-[5], [9], and [10], as a characterization of a geometric transformation under a mild hypothesis, which states that a bijection $\varphi$ mapping the set of lines of a hyperbolic projectivemetric plane to the set of lines of another hyperbolic projective-metric plane which satisfies $g \perp h \Leftrightarrow \varphi(g) \perp \varphi(h)$ is induced by an isomorphism of the two planes (i.e. a bijection defined on both the point- and the line-set of the plane, which preserves point-line incidence and line-orthogonality, as well as their negations).

Lingenberg's axiom system consists of the following assumptions (partly group-theoretic, partly geometric):

Let $G$ be a group generated by a subset $S$ consisting of involutions. The members of $S$ will be thought of as reflections in lines, and will be denoted by lowercase letters (which will also denote the lines themselves). Let $J$ denote the set of all involutions in $G$. We define the following notions for the group plane associated with $G$ : $\kappa$, standing for line-concurrence, by

$$
\kappa(a, b, c): \Leftrightarrow a b c \in J
$$

$\perp$, standing for line-orthogonality, by

$$
a \perp b: \Leftrightarrow a b \in J
$$

the notion of a point, defined, for any two different lines $a$ and $b$, as the set $S(a b):=\{x \mid a b x \in J\}$; and the notion of point-line incidence, a line $l$ being incident with the point $S(a b)$ if

$$
l \in S(a b) .
$$

A point $A$ is called:

(i) $\Delta$-connected if, for any three points $B, C, D$ which are mutually connected by lines, there is a line connecting $A$ to one of $B, C$ or $D$,

(ii) 1- $\Delta$-connected if it is $\Delta$-connected, and if there are three mutually connected points $B, C, D$ for which $A$ is connected with exactly one point of the triple,

(iii) 2- $\Delta$-connected if it is $\Delta$-connected but not $1-\Delta$-connected, and if there is a point to which it is not connected.

The group $G$ satisfies the following axioms (all lowercase letters refer to members of $S$ ):

(I) if $a \neq b$ and $a b x, a b y, a b z \in J$, then $x y z \in S$, 
(II) there exist $a, b, c, d$ such that none of $a b c, a c d, a b d, b c d$ is in $J$, and the group plane satisfies the axioms:

(III) every point is $\Delta$-connected,

(IV) there exist two distinct $2-\Delta$-connected points which are connected by a line,

(V) there exists a $1-\Delta$-connected point.

To express this axiom system in terms of $\perp$ alone, we shall first define the notion $\epsilon$, of end, as a pair of lines without a common perpendicular, i.e.

$$
\epsilon(a, b): \Leftrightarrow(\forall g) \neg(g \perp a \wedge g \perp b),
$$

by means of which we define $\kappa$ in terms of $\perp$ alone as follows (here and in the sequel the sum in the indices is $\bmod 3)$ :

$$
\begin{aligned}
\kappa\left(a_{1}, a_{2}, a_{3}\right): \Leftrightarrow & \left(a_{1}=a_{2} \wedge a_{2}=a_{3}\right) \\
& \vee\left\{\bigvee _ { i = 1 } ^ { 3 } \left[a_{i} \neq a_{i+1} \wedge\left(\left(a_{i+2}=a_{i} \vee a_{i+2}=a_{i+1}\right)\right.\right.\right. \\
& \vee\left((\exists h) \bigwedge_{j=1}^{3} a_{j} \perp h\right) \\
& \left.\left.\left.\vee\left((\exists d) \bigwedge_{k=1}^{3}\left(\epsilon\left(a_{k}, a_{k+1}\right) \wedge \epsilon\left(a_{k}, d\right)\right)\right)\right)\right]\right\} .
\end{aligned}
$$

This definition says that three lines, two of which are different, are concurrent if and only if the third one is equal to one of the other two, or they either have a common perpendicular or are all meeting in an end (i.e. in a point on the absolute). The line $d$ required to meet each of the $a_{i}$ in an end is there to prevent $a_{1}, a_{2}, a_{3}$ from meeting in pairwise different points.

Given that now $\kappa$ is an abbreviation for a formula in terms of $\perp$, we can define in terms of $\perp$ the predicate $\sigma$, with $\sigma\left(g, h, h^{\prime}\right)$ standing for $h^{\prime}$ is the reflection of $h$ in $g$, by

$$
\begin{aligned}
\sigma\left(g, h, h^{\prime}\right): \Leftrightarrow & \left((h \perp g \vee h=g) \wedge h^{\prime}=h\right) \vee(h \not \perp g \wedge h \neq g \\
& \wedge\left(\left(\exists i j i^{\prime}\right) i \perp h \wedge i \not \perp g \wedge \kappa(h, i, j) \wedge \neg \kappa(h, i, g) \wedge j \perp g\right. \\
& \left.\left.\wedge \kappa\left(h^{\prime}, i^{\prime}, j\right) \wedge h \neq h^{\prime} \wedge h^{\prime} \perp i^{\prime} \wedge \kappa\left(i, i^{\prime}, g\right) \wedge \kappa\left(h, h^{\prime}, g\right)\right)\right) .
\end{aligned}
$$

To see that the choice of $i, j, i^{\prime}$ is always possible in a hyperbolic projective-metric plane over a field with more than five elements, let us denote by $\pi(g)$ and $\pi(h)$ the poles of the lines $g$ and $h$. Given $g \not \perp h$, the two poles are on neither $g$ nor $h$. There are at most two lines in the projective plane passing through a pole which are self-polar, so there are altogether at most four lines passing through $\pi(g)$ and $\pi(h)$ which are not lines of the hyperbolic projective-metric plane. These four lines, as well as the line joining $\pi(g)$ and 


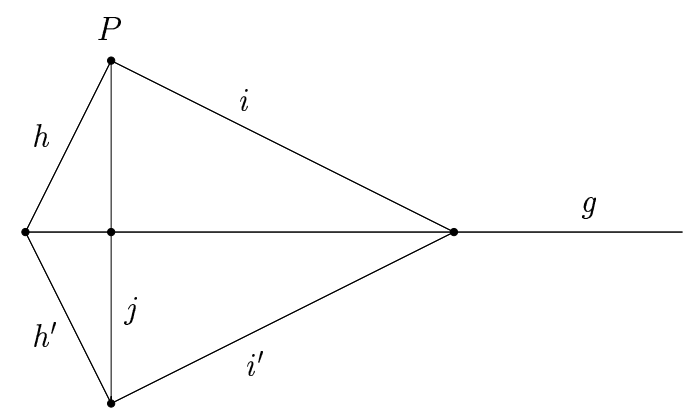

Fig. 1. $\sigma\left(g, h, h^{\prime}\right)$

$\pi(h)$, intersect $h$ in a set $S$ of at most five points. Since $h$ contains more than six points (as the coordinate field is neither $\mathrm{GF}(3)$ nor $\mathrm{GF}(5)$, nor can it be $\mathrm{GF}(4)$, because the coordinate field must have characteristic $\neq 2$ ), we can choose a point $P$ on it which is different from the intersection point of $g$ with $h$ and is not in $S$. Let $j$ be the line joining $P$ to $\pi(g)$, let $i$ be the one joining $P$ to $\pi(h)$, and let $h^{\prime}$ and $i^{\prime}$ denote the reflections of $h$ and $i$ in $g$. Since reflections preserve both incidence and orthogonality, the lines $g, h, h^{\prime}, i, j, i^{\prime}$ are as required by (1) in the case where $g$ and $h$ are neither equal nor perpendicular. It can be checked, given that line-orthogonality is equivalent to the condition that a symmetric bilinear form be zero, that for given $g, h, i, j$ with $h \not \perp g, i \perp h, i \not \perp g, j \perp g$, with $h, i, g$ not concurrent and $h, i, j$ concurrent, there is only one pair $\left(h^{\prime}, i^{\prime}\right)$ satisfying the conditions in the definition of $\sigma$.

Let $\varphi$ and $\psi$ be the sentences describing the hyperbolic projective-metric plane over GF(3) and GF(5) respectively in terms of $\perp$ (they state the exact number of lines, and specify all the orthogonalities that take and all those that do not take place among those lines).

We can easily reformulate the definitions of $\Delta$-connected, $1-\Delta$-connected, and 2 - $\Delta$-connected points in the language of lines (a point will be identified with the two lines determining it), with $\neq(x, y, z)$ standing for $x \neq y \wedge$ $y \neq z \wedge z \neq x$ :

$$
\begin{aligned}
\delta(a, b): \Leftrightarrow & a \neq b \wedge\left(\forall g_{1} g_{2} g_{3}\right)(\exists u) \neq\left(g_{1} g_{2} g_{3}\right) \wedge \neg \kappa\left(g_{1}, g_{2}, g_{3}\right) \\
\rightarrow & \kappa(a, b, u) \wedge\left(\bigvee_{i=1}^{3} \kappa\left(g_{i}, g_{i+1}, u\right)\right), \\
\delta_{1}(a, b): \Leftrightarrow & \delta(a, b) \wedge(\exists l m n v)(\forall w) \neq(l m n) \wedge \neg \kappa(l, m, n) \wedge \kappa(a, b, v) \\
& \wedge \kappa(l, m, v) \wedge \neg((\kappa(m, n, w) \vee \kappa(n, l, w)) \wedge \kappa(a, b, w)), \\
\delta_{2}(a, b): \Leftrightarrow & \delta(a, b) \wedge \neg \delta_{1}(a, b) \wedge[(\exists p q)(\forall v) p \neq q \wedge \neg(\kappa(a, b, v) \wedge \kappa(p, q, v))] .
\end{aligned}
$$

We can now state our axiom system for hyperbolic projective-metric planes 
over fields different from $\mathrm{GF}(3)$ or $\mathrm{GF}(5)$. The axioms are:

A1 $(\forall g h)\left(\exists^{=1} h^{\prime}\right) \sigma\left(g, h, h^{\prime}\right)$,

A2 $\sigma\left(g, h, h^{\prime}\right) \rightarrow \sigma\left(g, h^{\prime}, h\right)$,

A3 $\left(\exists a_{1} a_{2} a_{3} a_{4}\right) \quad \bigwedge \neg \kappa\left(a_{i}, a_{j}, a_{k}\right)$, $1 \leq i<j<k \leq 4$

A4 $(\forall a b x y z)(\exists u)(\forall m n p q) a \neq b \wedge \kappa(a, b, x) \wedge \kappa(a, b, y) \wedge \kappa(a, b, z)$

$\rightarrow(\sigma(z, m, n) \wedge \sigma(y, n, p) \wedge \sigma(x, p, q) \rightarrow \sigma(u, m, q))$,

A5 $a \neq b \rightarrow \delta(a, b)$,

A6 $(\exists a b c) \neg \kappa(a, b, c) \wedge \delta_{2}(a, b) \wedge \delta_{2}(a, c)$,

A7 $(\exists a b) \delta_{1}(a, b)$,

A8 $\quad(\forall a b c) \kappa(a, b, c) \leftrightarrow\left((\forall x)\left(\exists x^{\prime} y y^{\prime} z z^{\prime}\right) \sigma\left(c, x, x^{\prime}\right) \wedge \sigma\left(b, x^{\prime}, y\right) \wedge \sigma\left(a, y, y^{\prime}\right)\right.$ $\left.\wedge \sigma\left(c, y^{\prime}, z\right) \wedge \sigma\left(b, z, z^{\prime}\right) \wedge \sigma\left(a, z^{\prime}, x\right)\right)$,

A9 $(\forall a b) a \perp b \leftrightarrow\left((\forall x)\left(\exists x^{\prime} y y^{\prime}\right) \sigma\left(b, x, x^{\prime}\right)\right.$

$\left.\wedge \sigma\left(a, x^{\prime}, y\right) \wedge \sigma\left(b, y, y^{\prime}\right) \wedge \sigma\left(a, y^{\prime}, x\right)\right)$,

as well as an axiom $\alpha$ stating that there are at least 28 different lines. We think of all the axioms as stated with the perpendicularity notion alone, i.e. we think of the notions defined as having been replaced by their definiens.

Given that, by A1, for any line $g$ we can define a map $\sigma_{g}$ which maps every line $h$ into the line $h^{\prime}$ for which $\sigma\left(g, h, h^{\prime}\right)$, we notice that: A2 states that the $\sigma_{g}$ are involutory, A8, A9 ensure that (*) and (**) hold, A4 implies axiom (I) for the group $G$ generated by the subset $S$ consisting of the mappings $\sigma_{g}$, A3 implies (II), A5 implies (III), A6 implies (IV), and A7 implies $(\mathrm{V})$. Given that the $\sigma_{g}$, which are involutory, satisfy the axiom system (I)-(V) (with the notions of line-concurrence, orthogonality, and point-line incidence defined by $(*),(* *)$, and $(\dagger))$, they must generate the motion group of a hyperbolic projective-metric plane. Given A9, $\perp$ has the desired interpretation. Thus if we designate by $\zeta$ the conjunction of the axioms $\alpha$ and A1-A9, then $\varphi \vee \psi \vee \zeta$ axiomatizes hyperbolic projective-metric planes.

Replacing A6 in the above axiom system with

$\mathrm{A} 10 \quad(\forall a)(\exists b c) \neg \kappa(a, b, c) \wedge \delta_{2}(a, b) \wedge \delta_{2}(a, c)$,

we obtain an axiom system, consisting of A1-A5, A7-A9, and A10, for hyperbolic projective-metric planes over quadratically closed fields of characteristic $\neq 2$. These planes were also called hyperbolic-metric planes with free mobility in [8], where they were characterized in terms of motions.

Using the representation theorem 6.25 from [7], we have proved:

THEOREM. Every model of $\varphi \vee \psi \vee \zeta$ is isomorphic to the set of lines of a hyperbolic projective-metric coordinate plane coordinatized by a field 
of characteritic $\neq 2$, and $\perp$ is interpreted as line-orthogonality. The same conclusion, with quadratically closed fields of characteristic $\neq 2$ as coordinate fields, holds for all models of A1-A5, A7-A9, and A10.

\section{References}

[1] F. Bachmann, Aufbau der Geometrie aus dem Spiegelungsbegriff, 2. Auflage. Springer, Berlin, 1973.

[2] W. Benz, Geometrische Transformationen, BI Wissenschaftsverlag, Mannheim, 1992.

[3] W. Benz und E. M. Schröder, Bestimmung der orthogonalitätstreuen Permutationen euklidischer Räume, Geom. Dedicata 21 (1986), 265-276.

[4] H. Havlicek, On Plücker transformations of generalized elliptic spaces, Rend. Mat. Appl. (7) 15 (1995), 39-56.

[5] -, A characteristic property of elliptic Plücker transformations, J. Geom. 58 (1997), 106-116.

[6] R. Lingenberg, Vollständige metrische Ebenen, Abh. Math. Sem. Univ. Hamburg 48 (1979), 241-263.

[7] —, Metric Planes and Metric Vector Spaces, Wiley, New York, 1979.

[8] —, Hyperbolisch-metrische Ebenen mit freier Beweglichkeit, in: Symposia Mathematica 11 (Convegno di Geometria, INDAM, Rome, 1972), Academic Press, London, 1973, 397-412.

[9] K. List, On orthogonality-preserving Plücker transformations of hyperbolic spaces, Abh. Math. Sem. Univ. Hamburg 70 (2000), 63-75.

[10] —, Harmonic mappings and hyperbolic Plücker transformations, J. Geom. 70 (2001), $108-116$.

[11] V. Pambuccian, Aufbau der hyperbolischen Geometrie aus dem Geradenorthogonalitätsbegriff, Acta Math. Hungar. 101 (2003), 51-61.

[12] - Hyperbolic geometry in terms of point-reflections or of line-orthogonality, Math. Pannon., to appear.

[13] V. Pambuccian, K. Prażmowski and K. Sakowicz, Defining co-punctuality in terms of line-orthogonality in plane hyperbolic geometry, Acta Math. Hungar., submitted.

[14] K. Sakowicz, Axiomatizability of the dual external hyperbolic plane, Master's thesis, Warsaw University, Białystok Branch, 1988 (in Polish).

Victor Pambuccian

Department of Integrative Studies

Arizona State University West

P.O. Box 37100

Phoenix, AZ 85069-7100, U.S.A.

E-mail: pamb@math.west.asu.edu

Received December 9, 2003;

received in final form February 11, 2004 